\title{
A controlled trial of a low sodium, low fat, high fibre diet in treated hypertensive patients: the efficacy of multiple dietary intervention
}

\author{
Paul Little, ${ }^{1}$ Gillian Girling, ${ }^{2}$ Anne Hasler, ${ }^{2}$ Sir Anthony Trafford ${ }^{1}$ and Arthur \\ Craven $^{3}$
}

\author{
${ }^{1}$ Hypertension Clinic, Royal Sussex County Hospital, ${ }^{2}$ Department of Nutrition and Dietetics, Royal \\ Sussex County Hospital, Eastern Road, Brighton, East Sussex BN2 5BE, and ${ }^{3}$ Department of \\ Mathematics, University of Sussex, Falmer, East Sussex, BN1 9QH, UK.
}

\begin{abstract}
Summary: An 8-week combination low sodium, low fat, high fibre diet was compared to the individual components of this diet in a controlled trial using 193 patients already on treatment for essential hypertension. No significant changes were observed in the high fibre group. The low sodium group showed a decrease in systolic blood pressure which was close to significance. The low fat group showed a small but significant decrease in seated diastolic blood pressure and weight. The combination group showed larger and highly significant decreases in seated and standing systolic blood pressures, seated diastolic blood pressure and weight, as well as a significant reduction in standing diastolic blood pressure.

We conclude that multiple dietary intervention in this case in the form of a low sodium, low fat, high fibre diet, is more effective than any single dietary intervention and is useful in patients already on medication.
\end{abstract}

\section{Introduction}

Numerous dietary factors have been suggested as alternatives to drug therapy in the management of hypertension. Comparing a number of studies a low sodium diet has a moderate effect; ${ }^{1}$ potassium supplementation, ${ }^{2}$ a low fat intake ${ }^{3}$ and a vegetarian diet, possibly secondary to the high fibre content, ${ }^{4,5}$ have also been shown to reduce blood pressure. A combination of these factors in a low sodium, low fat, high fibre diet was then shown to be effective in lowering blood pressure in diabetic subjects $^{6-8}$ and in treated hypertensive patients. ${ }^{9,10}$ However, it is not clear if a combination of dietary factors reduces blood pressure more effectively than any of the individual components. Furthermore, it is uncertain to what extent dietary factors are useful in combination with anti-hypertensive medication since most patients in these studies were not receiving treatment for hypertension, and one of the studies in treated patients was uncontrolled, ${ }^{9}$ although a more recent study was controlled. ${ }^{10}$

\footnotetext{
Correspondence: P. Little B.A., M.B., B.S., M.R.C.P. c/o The Yews, Lews Road, Ringmer, East Sussex BN8 5ET Lord Trafford (Sir Anthony Trafford) sadly and unexpectedly died since this study commenced. Most patients in this study were picked up by the Royal Sussex County Hospital hypertension clinic and screening survey, which, with the renal unit, were set up largely by Lord Trafford's effort.

Accepted: 16 February 1990
}

Finally there is up to $80 \%$ variation in quantitative assessment using different methods in weil motivated subjects ${ }^{11}$ and many of the methods of assessment, with the exception of the food frequency interview, ${ }^{11}$ are not used in routine clinical practice.

We have therefore assessed the effect of a low sodium, low fat, high fibre diet compared to the individual components of this diet in patients already receiving drug treatment for hypertension, using a food frequency interview to assess compliance.

\section{Subjects and methods}

The 193 patients studied (aged 30-71) were already on treatment for hypertension under the care of the Royal Sussex County Hospital Hypertension Clinic or their general practitoners.

Patients' blood pressure and pulse measurements were taken by one observer on three occasions initially at weekly intervals, using the third visit values as the baseline, and after 8 weeks in each of the study groups. An average of 3 readings of both seated blood pressure after 5 minutes and standing blood pressure after 30 seconds was taken using a Hawksely random zero sphygmomanometer. Diastolic blood pressure was measured to the 5th Korotkoff phase. One patient 
(in group C) required a large cuff. Since most patients' initial blood pressures were well controlled, the expected changes with dietary intervention were small. Thus particular attention was paid to reducing the variability in blood pressure readings. As many patients showed a fall in blood pressure within each clinic visit, to reduce the variability resulting from this effect, the last 3 of 6 repeated measurements were taken. To limit the artifact of anxiety if patients spontaneously expressed particular worries they were asked to return a week later (this only applied to 3 patients in group E, 1 in group A, and 1 in group D for the week 8 visit). Following the blood pressure readings a seated pulse measurement after 30 seconds was taken.

Dietary assessment was by a food frequency interview ${ }^{11}$ closely corresponding to the assessment of routine clinical practice: this consisted of a detailed and general history concerning the level and frequency of consumption of all foods and drink lasting approximately $\frac{3}{4}-1$ hour, which, combined with information from McCance and Widdowson's food tables, ${ }^{12}$ allowed a full qualitative and quantitative picture of the patient's diet. Since urinary sodiums were not used, discretionary sodium (i.e. added at the table or in cooking) was estimated using average UK figures, that is approximately $12-16 \%$ of the total, ${ }^{13-15}$ combined with a simple patient estimation of low ('seldom or never'), moderate ('if needed after tasting'), or high ('routinely before tasting') addition. There is a relatively good correlation (correlation coefficient 0.65 ) between patient estimation and recorded intake using this method and patient variability is low allowing an acceptable relative measurement of sodium intake. ${ }^{16}$

After initial dietary assessment and weight measurement (using Marsden balanced scales), patients were allocated sequentially to one of the following 5 study groups by the dietician thus keeping the observer blind:

Group A - control: no change in diet $(n=40)$

Group B - high fibre diet $=40-45 \mathrm{~g}$ soluble and insoluble fibre per day $(n=38)$.

Group C-low sodium diet $=40-50 \mathrm{mmol} \mathrm{Na}^{+}$ per day $(n=33)$.

Group D-low fat diet $=23-25 \%$ calories as fat per day with no change to the polyunsaturated $(\mathrm{P}) /$ saturated $(\mathrm{S})$ or monounsaturated $(\mathrm{M}) / \mathrm{S}$ ratios $(n=41)$.

Group E - combination diet: low sodium, low fat, high fibre diet $=40-45 \mathrm{~g}$ fibre, $40-50 \mathrm{mmol} \mathrm{Na}{ }^{+}, 23-25 \%$ calories as fat per day $(n=41)$.

The dietary objectives in each group were based on the combination diet used in previous studies. ${ }^{6-10}$ Patients were asked not to change alcohol consumption or smoking habits during the trial. Patients already following one of the proposed diets were excluded from that group. Patients with renal impairment (creatinine $>150 \mu \mathrm{mol} / \mathrm{litre}$ ) and patients whose medication had been altered during the previous 2 weeks or during the study were excluded. These factors together with patient drop-out, caused a discrepancy in numbers in different groups (see Table I). The patient characteristics for each group are shown in Table II.

The patients were followed up for monitoring and assessment of compliance by the dieticians (with further food frequency interviews) at week 4 and week 8 of the diet. The compliance assessment categories for groups B-D are shown in Table III. For group $E$ an average of the ratings for individual components was taken. Seventy five per cent of patients were seen initially by one dietician (GG) who also saw all but $2 \%$ of the patients at some time in the study, and liaised closely with the other dieticians involved, thus increasing the comparability of the assessments. At the end of the study patients were asked whether they found their diet unpalatable, moderately palatable, or palatable. Results were analysed by Student's $t$ test. The relationship between blood pressure changes and weight changes were analysed by Pearson's correlation coefficient.

\section{Results}

There were no significant changes in pulse in any of the groups. The changes in seated and standing blood pressure and weight measurements after an 8-week period in the control group and the changes in the various dietary groups relative to the control group and are shown in Table IV.

The high fibre group did not show any significant changes in blood pressure. The low sodium group showed a modest decrease in seated systolic pressure $(P<0.07)$ and a significant decrease in weight $(P<0.05)$ when compared to the control group. Small but significant decreases in seated diastolic blood pressure $(P<0.05)$ and weight $(P<0.01)$ occurred in the low fat group; the significant

Table I Allocation and exclusion of patients

\begin{tabular}{|c|c|c|c|c|c|}
\hline & \multicolumn{5}{|c|}{ Group } \\
\hline & $A$ & $\boldsymbol{B}$ & $C$ & $D$ & $E$ \\
\hline Initial allocation & 45 & 40 & 36 & 42 & 44 \\
\hline \multicolumn{6}{|l|}{ Exclusion: } \\
\hline Medication changed & 3 & & & & 1 \\
\hline $\begin{array}{l}\text { Renal impairment } \\
\text { (creatinine }>150 \mu \mathrm{mol} / \text { litre) }\end{array}$ & 1 & 1 & & & \\
\hline Did not attend for follow-up & 1 & 1 & & 1 & 2 \\
\hline Final number & 40 & 38 & 33 & 41 & 41 \\
\hline
\end{tabular}


Table II Characteristics of patients in different study groups

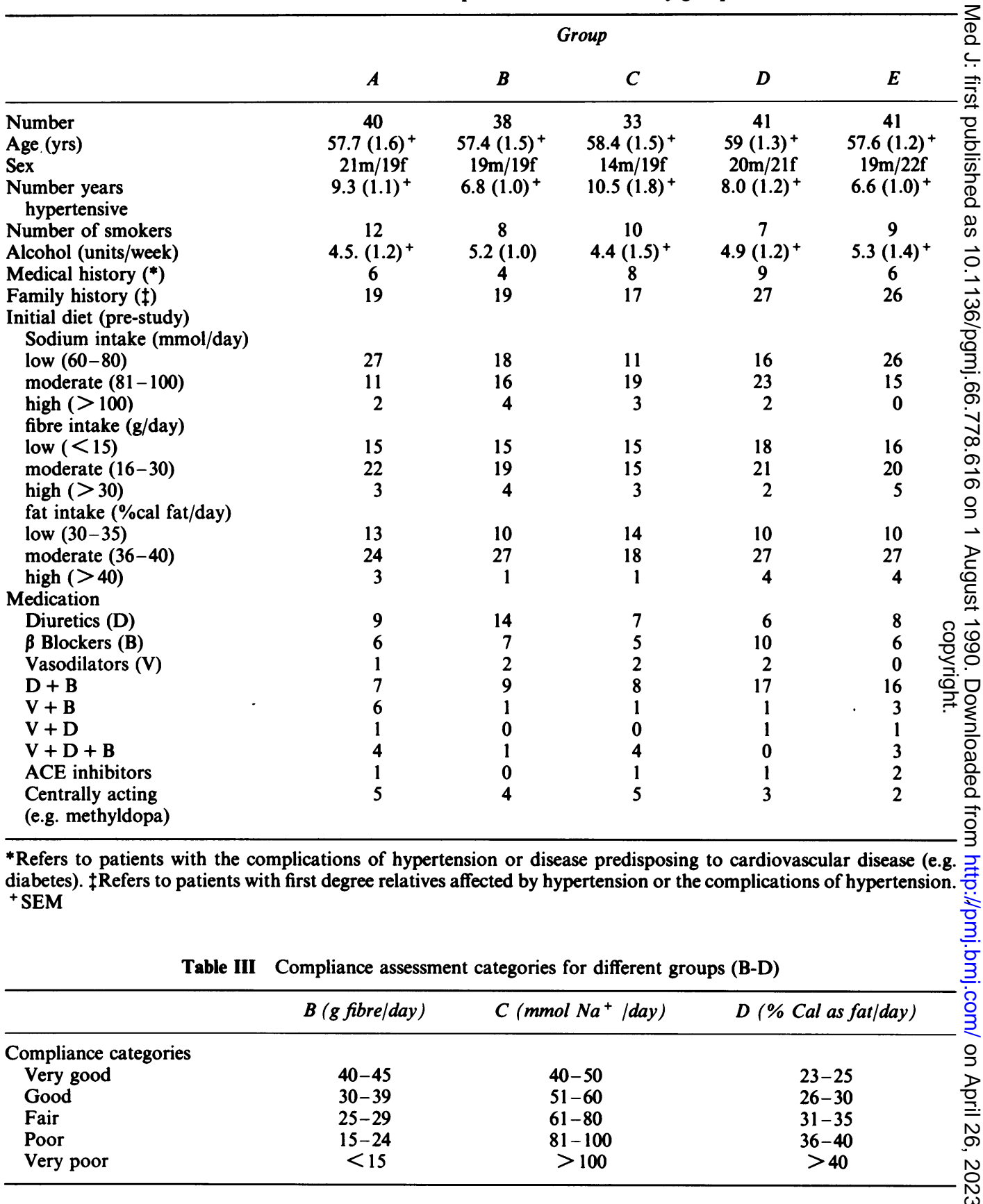

change in diastolic pressure was as much due to a small rise in diastolic pressure in the control group. The combination low sodium, low fat, high fibre group showed larger and highly significant decreases in seated systolic $(P<0.001)$, seated diastolic $(P<0.001)$, standing systolic $(P<0.001)$ blood pressures and weight $(P<0.001)$, as well as a significant change in standing diastolic blood pressure $(P<0.05)$. The changes of blood pressure and ${ }_{\overparen{D}}$ weight in the combination group were significantly $\stackrel{?}{?}$ larger than in any single group $(P<0.05)$. There 0 was no correlation between weight changes and $\bar{P}$ blood pressure changes across all the groups (correlation coefficient $\mathbf{0 . 1}$ ). 
Compliance at week 8 and palatability assessments after the end of the study are shown in Table V. Only 3 patients in group A made any change to their diet. Compliance was generally good, with the majority of patients in the good or very good categories in all groups. Similarly, most patients found the diets palatable or moderately palatable. Within this context compliance and palatability ratings were worst for the combination diet, followed by the low salt diet and the low fat diet, with the high fibre diet achieving the best ratings.

\section{Discussion}

Two main findings emerge in this study. Firstly, single dietary modifications in treated hypertensive patients cause modest changes in blood pressure consistent with those observed in untreated hypertensives. ${ }^{1-3}$ The exception appears to be dietary fibre: this group showed a change in blood pressure over the study period comparable to the control group. Differences in protocol may partly account for this; for example Wright et al ${ }^{4}$ used patients with a low fibre diet whereas some of the patients in the high fibre group on this study already had moderate fibre intake. Also dietary fibre may differ in its effect in treated hypertensive patients in that drug absorbtion may be altered. Furthermore, a trial of fibre in normotensive volunteers showed no effect on blood pressure; ${ }^{13}$ the relevance of this to hypertensive patients is unclear, but it does question the role of dietary fibre in blood pressure. The relatively small nonsignificant effect seen with the low salt group may well be an underestimate in view of the fact that many patients had restricted their salt intake to some extent (see Table II).

The second important finding is that a combination of dietary interventions in a low sodium, low fat, high fibre diet causes a greater fall in both systolic and diastolic blood pressures than any of those interventions alone. This effect cannot be explained by compliance since compliance was marginally worse in the combination group (see Table V). What is not clear is whether a low sodium, low fat diet would be as effective as a low sodium, low fat, high fibre diet, since a high fibre diet alone was not effective in this group of patients.

A major issue with dietary intervention is palatability and compliance. Even with well motivated patients there is wide and often systematic variation in quantitative assessment using computerized food tables and different types of dietary history; ${ }^{11}$ it is not clear if this is due to inherent inaccuracy of the methods, or an effect on compliance, or both. Two issues follow from this: firstly the accuracy of quantitative assessment must 
Table V Compliance and palatability rating for the different groups (see Table II for compliance assessment categories)

\begin{tabular}{llrrrr}
\hline & \multicolumn{4}{c}{ Group } \\
& & $B(\mathrm{n}=38)$ & $C(\mathrm{n}=33)$ & $D(\mathrm{n}=41)$ & $E(\mathrm{n}=41)$ \\
\hline Compliance & Very good & 18 & 9 & 12 & 8 \\
& Good & 14 & 12 & 19 & 17 \\
& Fair & 5 & 9 & 7 & 12 \\
& Poor & & 3 & & 3 \\
& Very poor & & & 3 & 1 \\
Not assessed & 1 & & & \\
Palatability & Not palatable & 5 & 12 & 3 & 18 \\
& Moderately palatable & 4 & 12 & 20 & 10 \\
& Palatable & 29 & 9 & 18 & 13 \\
\hline
\end{tabular}

inevitably be limited, hence an emphasis on the detailed nature of the dietary history to ensure at least a good qualitative assessment. Secondly, the method of compliance assessment may affect compliance. Thus in this study a food frequency interview was used, most closely corresponding to the assessment of routine clinical practice, performed by dieticians as part of their normal clinical work in a busy district general hospital, with the result that the blood pressure changes should represent what is possible in normal clinical practice. A similar argument applied to the use of urinary sodium measurements since they are not used in routine clinical practice. In addition, in this particular study since the main aim was to compare numerous dietary factors on an equal basis, it was felt important not to give any one dietary factor a different assessment bias and hence possibly different compliance. Furthermore, the main aim of comparing compliance to the various dietary components would not be helped by a separate more accurate method of assessment for just one component such as sodium. The disadvantage of the method used is that comparability of sodium intake with other studies is difficult, and less accurate in view of the difficulty of estimating salt added at the table and in cooking, although the error is probably less in this group of patients since many had already reduced their discretionary sodium intake. In absolute terms the figures quoted in this study probably represent a relative underestimate: Dodson et al..$^{10}$ using a similar group of patients found urinary sodiums of $90 \mathrm{mmol} /$ day compared to an estimated $70 \mathrm{mmol}$ in both the low sodium and the combination groups in this study. Compliance assessment is limited by patient honesty; however, it was emphasized that honesty with the dieticians was more important than strict compliance. Compliance was possibly increased by motivating patients with a promised reduction in antihypertensive medication if blood pressure was well controlled at the end of one study. Many patients found the combination diet unpalatable and difficult to follow initially, but then gradually became accustomed to it. Dodson et al., using the combination diet,' found that long term compliance over a 4-year period was surprisingly good and that blood pressure changes were well maintained; similarly Stamler et al. using sodium, alcohol, and weight reduction, found that blood pressure changes were well maintained at 4 years. ${ }^{18}$

The mechanism of benefit of the combination diet is uncertain. Part of the effect may be through weight loss since larger and highly significant changes in weight were observed in this group compared to other groups. The relationship between weight loss and hypertension is generally accepted although when many studies are compared at least part of this effect may be due to sodium restriction. ${ }^{19}$ In diabetic patients on the combination diet significant changes in blood pressure have been observed without significant changes in weight, ${ }^{6}$ though subsequent studies did show significant changes in weight. ${ }^{7.8}$ However, there was no correlation between blood pressure and weight changes. In this study there was no straightforward connection between weight loss and changes in blood pressure as shown by a very low correlation coefficient $(0.1)$.

If a combination of factors is more effective than a single factor, what further dietary suggestions can be made in order to lower blood pressure? Although a low fat diet generally is beneficial, a mackerel diet rich in ecosapentanoic (EPA) and docosahexanoic acids (DHA) reduced both systolic and diastolic blood pressure in male patients with essential hypertension, ${ }^{20}$ and in a double-blind study MAXEPA, a fish oil rich in both EPA and DHA, was shown to be effective in both normal volunteers and patients on haemodialysis in reducing systolic blood pressure. ${ }^{21}$ Similarly, a doubleblind study showed that linoleic acid supplement causes a significant reduction in systolic blood pressure in normotensive volunteers, ${ }^{22}$ although 
the change is small. Potassium supplementation reduces blood pressure, ${ }^{2}$ as does calcium supplementation in about half of patients with essential hypertension, ${ }^{23,24}$ and magnesium supplementation is not only effective in lowering blood pressure in hypertensive encephalopathy but also in patients with essential hypertension. ${ }^{25}$ Increased calcium and magnesium in the diet may be partly responsible for the beneficial effect of a vegetarian diet. ${ }^{26}$ However, until the evidence for calcium and magnesium is more consistent, routine supplementation in patients with essential hypertension is not indicated. Thus probably the most beneficial diet for patients with essential hypertension would be not only a low sodium, low fat, high fibre diet, but also a diet rich in potassium, and in specific polyunsaturated fatty acids (EPA, DHA).

We conclude that multiple dietary intervention in routine clinical practice using a low sodium, low fat, high fibre diet is more effective than any single intervention in lowering blood pressure and is useful in those already on treatment.

\section{Acknowledgement}

Grateful thanks to Janet Queen for preparation of the manuscript.

\section{References}

1. Grobbee, P.E. \& Hofman, A. Does sodium restriction lower blood pressure? Br Med J 1986, 293: 27-29.

2. MacGregor, G.A., Smith, S.J., MacKandu, H.P., Banks, R.A. \& Sagnella, L.A. Moderate potassium supplementation in essential hypertension. Lancet 1982, i: 567.

3. Puska, P., Iancono, J., Nissinen, A. et al. Controlled randomised trial of the effect of dietary fat on blood pressure. Lancet 1983, i: 1-5.

4. Wright, A., Burstyn, P.C. \& Gibney, M.J. Dietary fibre and blood pressure. Br Med J 1979, 2: 1541-1543.

5. Rouse, I.G., Beilin, L.J., Armstrong, B.K. \& Vandongen, R. Blood pressure lowering effect of a vegetarian diet: controlled trial in normotensive subjects. Lancet 1983, i: 5 .

6. Dodson, P.M., Beevers, M., Fletcher, R.F., Pacy, P.J., Bal, P. \& Taylor, K.G. The effect of a high fibre, low fat and low sodium dietary regime on diabetic hypertensive patients of different ethnic groups. Postgrad Med J 1983, 59: 641-644.

7. Pacy, P.J., Dodson, P.M., Kubicki, A.J., Fletcher, R.F. \& Taylor, K.C. Effect of a high fibre, high carbohydrate dietary regime on serum lipids and lipoproteins in Type II hypertensive diabetic patients. Diabetes Res 1984, 1: 159-163.

8. Dodson, P.M., Pacy, P.J., Bal, P., Kubicki, A.J., Fletcher, R.F. \& Taylor, K.G. A controlled trial of a high fibre, low fat, and low sodium diet for mild hypertension in Type 2 (non insulin - dependent) diabetic patients. Diabetologia 1984, 27: $522-526$.

9. Dodson, P.M., Pacy, P.J. \& Cox, E.V. Long term follow up of the treatment of essential hypertension with a high fibre, low fat, low sodium dietary regime. Hum Nutr Clin Nutr 1985, 39C: $213-220$.

10. Dodson, P.M., Stephenson, J., Dodson, L.J. et al. Randomised blind control trial of a high fibre, low fat, low sodium dietary regimen in mild essential hypertension. $J$ Hum Hypertens 1989, 3: 197-202.

11. Bull, M.L. \& Wheeler, E.F. A study of different dietary survey methods among 30 civil servants. Hum Nutr Appl Nutr 1986, 40a: 60-66.

12. Paul, A.A. \& Southgate, D.A.T. (eds) McCance \& Widdowson's The Composition of Foods. HMSO, London, 1978.

13. Sanchez-Castillo, C.P., Warrender, S., Whitehead, T. \& James, W.P. Epidemiological assessment of sodium sources in the diet by use of the lithium marker technique. Proc Nutr Soc 1984, 43: 153a.
14. Shepherd, R.E., Farleigh, C.A \& Land, D. Effects of taste sensivity and preference on salt intake. Proc Nutr Soc 1984, 43: 87a.

15. Bull, N.L. \& Buss, D.H. Contribution of foods to sodium intake. Proc Nutr Soc 1980, 39: 30a.

16. Kuminiyika, S.K. \& Jones, D.Y. Patterns of week to week table salt use by men and women consuming constant diets. Hum Nutr Appl Nutr 1983, 37a: 348-356.

17. Margetts, B.M., Beilin, L.J., Vandongen, R. \& Armstrong, B.K. A randomised controlled trial of the effect of dietary fibre on blood pressure. Clin Sci 1987, 72: 343-350.

18. Stamler, R., Stamler, J., Grimm, R. et al. Nutritional therapy for high blood pressure. Final report of a four year randomised controlled trial - The hypertension control program. JAMA 1987, 257: 1484-1491.

19. Kaplan, N.H. Non drug treatment of hypertension. Ann Intern Med 1985, 102: 359-373.

20. Singer, P., Wirth, M., Voigt, S. et al. Blood pressure and lipid lowering effect of mackerel and herring diet in patients with mild essential hypertension. Atherosclerosis 1985, 56: 223-225.

21. Norris, P.J., Jones, C.J.H. \& Weston, M.J. Effects of dietary supplementation with fish oil on systolic blood pressure in mild essential hypertension. Br Med J 1986, 243: 104-105.

22. Heagarty, A.M., Ollerenshaw, J.D., Robertson, P.I., Bing, R.F. \& Swales, J.D. Influence of dietary linoleic acid on leucocyte sodium transport and blood pressure. $\mathrm{Br}$ Med J 1986, 293: 295-297.

23. Resnick, L., Nicholson, J.P. \& Laragh, J.M. Outpatient therapy of essential hypertension with dietary calcium supplementation (abstract). Clin Res 1984, 3: 616.

24. McCarron, D.A. \& Morris, C. Oral Ca in mild to moderate hypertension: a randomised placebo-controlled trial (abstract). Clin Res 1984, 32: 335A.

25. Dyckner, T. \& Wester, P.O. Effect of magnesium on blood pressure $\mathrm{Br}$ Med J (Clin Res) 1983, 286: 1847-1849.

26. Margetts, B.M., Beilin, L.J., Vandongen, R. \& Armstrong, B.K. Vegetarian diet in mild hypertension: a randomised controlled trial. Br Med J 1986, 293: 1468-1471. 